\title{
A Classification of Centres of Maximal Balls in $\mathbb{Z}^{3}$
}

\author{
Robin Strand \\ Centre for Image Analysis, Uppsala University, \\ Lägerhyddsvägen 3, SE-75237 Uppsala, Sweden \\ robin@cb.uu.se
}

\begin{abstract}
A classification of centres of maximal balls (CMBs) in $\mathbb{Z}^{3}$ derived from generalizations of the chessboard and city block metrics to $3 \mathrm{D}$, a weighted metric, and the Euclidean metric is presented. Using these metrics, the set of CMBs (the medial axis) can be extracted. One difficulty with skeletonization in $3 \mathrm{D}$ is that of guaranteeing reversibility. A reversible skeleton generally consists of both surfaces and curves. Previous attempts to construct connected skeletons including the CMBs uses conditions based on local neighbourhood configurations. However, a local neighbourhood might be too small and, most important, does not allow a consistent definition for surface- and curve-parts of the skeleton. The classification of the CMBs presented in this paper will be a tool for defining which parts of a $3 \mathrm{D}$ skeleton are surfaces and curves.
\end{abstract}

\section{Introduction}

The medial axis transform - the detection of centres of maximal balls (CMBs) in a $2 \mathrm{D}$ binary shape - was proposed by Blum, [1]. A ball included in an object is a maximal ball if it is not completely covered by any other singel ball also included in the object. The CMBs can be used to construct reversible skeletons. Skeletons are widely used in image analysis within many applications. Many different approaches for constructing skeletons have been developed.

The set of CMBs is a thin connected set in $\mathbb{R}^{3}$, but in $\mathbb{Z}^{n}$ it is in general neither thin nor connected. To construct a connected digital skeleton representing the original object, these CMBs have to be connected. One approach to construct a connected skeleton was presented in [2]. With this approach, a reversible skeleton, i.e., a thin, centered, and topologically equivalent representation of the object from which the original object can be reconstructed, is generated. The set of CMBs are anchor-points, i.e., voxels that are not allowed to be removed. The rules are designed to connect the CMBs in such a way that the resulting skeleton consists of surfaces and curves. These rules are usually based on local configurations of the object. The problem is that there is no definition of "surface" or "curve" skeletal voxels. Basically, the rules check a local neighbourhood of the voxels and from that voxel configuration decides if the voxel should belong to the skeleton as a part of a surface or a curve, or be assigned to the background. 
A local neighbourhood might, however, not be sufficient; CMBs corresponding to "surface"-parts of the object can be located at a larger distance from any other CMB than what can be detected by a local neighourhood.

A classification of the skeletal points (the CMBs) in $\mathbb{R}^{3}$ into sheets, curves, and points has been made recently, [3]. The formal classification is based on the number of tangencies and the order of the tangency for each maximal ball. The classes are denoted $A_{k}^{n}$, where $n$ is the number of tangencies and $k$ is the order of the tangency between the border of the maximal ball and the border of the object. Using this approach, each CMB can be classified as belonging to a sheet, a curve, or a point part of the skeleton.

- Sheets consist of skeletal points where the maximal balls have two distinct tangencies, i.e., $A_{1}^{2}$ points.

- Curves can be divided into intersection curves of three sheets, $A_{1}^{3}$, and the boundary of sheets, i.e., $A_{3}$ points (higher order tangency points).

- Points are either centers of quad-tangent spheres, $A_{1}^{4}$, or centers of balls with one regular tangency and one higher order tangency, $A_{1} A_{3}$.

In Figure 1(a), an elliptic cylinder with two planar ends in $\mathbb{R}^{3}$ is shown. The medial axis representation (set of CMBs) of the cylinder is shown in Figure 1(b). The CMBs have been divided into sheets, curves, and points according to the classification above, see Figure 1(c) for a classification of the flat sheet (the middle part of the medial axis representation).

In this paper, $\mathbb{Z}^{3}$ equipped with four different metrics is considered; the simple metrics $D^{6}$ and $D^{26}$, a weighted metric based on the weights 3 , 4, and 5 , and the Euclidean metric. The CMBs are computed and classified based on the number of detected regions where the balls touch the border of the object. Classes similar to the classes previously defined in $\mathbb{R}^{3}$ are thus now defined in $\mathbb{Z}^{3}$.

\section{Basic Concepts and Notations}

Two voxels in $\mathbb{Z}^{3}$ are 6-adjacent if they share a face and 26-adjacent if they share at least a vertex. Adjacent voxels will also be denoted neighbours. A set of voxels is 6 -connected if there is a path consisting of pairwise 6 -adjacent voxels in the set between any two voxels in the set. The definition of 26 -connected sets is analogous. A binary image $I$ consists of two sets, the set of object voxels $X$ and the set of background voxels $\bar{X}$. In this paper, $X$ is assumed to be 26 -connected. The border of $X$ consists of the voxels in $X$ 6-adjacent to a voxel in $\bar{X}$.

The distance between two voxels using the metrics $D^{6}, D^{26}$, or $\left.<3,4,5\right\rangle$ is defined as the shortest path between the voxels allowing only adjacent voxels, [4]. With unit distance between adjacent voxels, the resulting distance is based on the metrics $D^{6}$ and $D^{26}$, using 6-adjacency and 26-adjacency, respectively. To get the distance between two voxels using the metric $<3,4,5>$, the local 


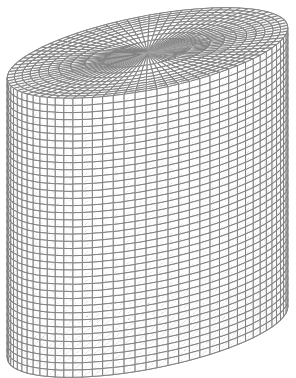

(a)

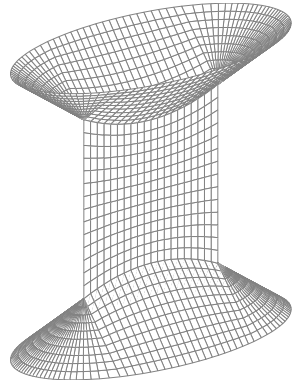

(b)

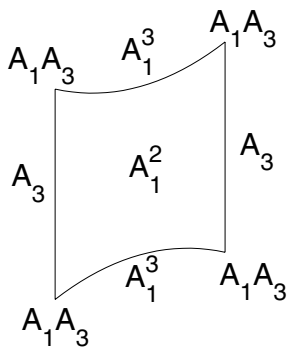

(c)

Fig. 1. An elliptic cylinder $\mathbb{R}^{3}$ with two planar ends (a), its medial axis representation (b), and the classification of the flat sheet in the middle of the medial axis representation (c)

distance between face, edge, and vertex neighbours are weighted with 3,4 , and 5 , respectively. With this definition, it is obvious that the triangle inequality is fulfilled. It follows that the distance functions are metrics. The digital Euclidean metric is defined using the usual Euclidean distance between the centers of the voxels. A ball is denoted $B(x, r)=\{y: d(x, y)<r)\}$, where $d(x, y)$ is the distance between $x$ and $y$ using $D^{6}, D^{26},<3,4,5>$, or the Euclidean metric.

When computing the distance transform (DT), each voxel in $X$ is assigned a label corresponding to the distance from the voxel to the closest voxel in $\bar{X}$. The DTs based on the metrics $D^{6}, D^{26}$, and $<3,4,5>$ are computed by propagating distance information locally in a two-scan Chamfer algorithm and are denoted $D T^{6}, D T^{26}$, and $W D T$. To compute the Euclidean DT $(E D T)$, four scans are needed, [5]. Using this method, small errors in the EDT will be produced. For an error-free algorithm, see, e.g., [6]. In Figure 2, balls of radius 18 (18 3 for $W D T)$ using the different metrics are shown. See $[5,4]$ for further information about DTs in 3D images.

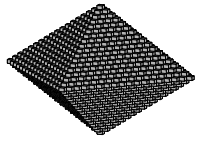

(a)

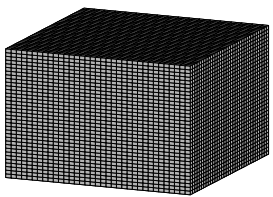

(b)

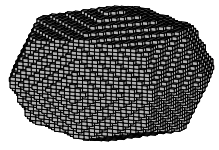

(c)

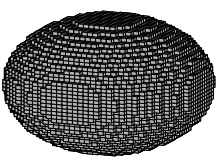

(d)

Fig. 2. Balls in $\mathbb{Z}^{3}$ using $D^{6}$ (a), $D^{26}$ (b), $<3,4,5>$ (c), and the Euclidean (d) metrics 


\section{$3 \quad$ Extracting the CMBs}

Using $D T^{6}$ and $D T^{26}$, the CMBs are easy to identify. In the same way as for the city block and chessboard metrics in $\mathbb{Z}^{2}$, they appear as local maxima in the DTs, [7]. A voxel in $X$ is a CMB if none of the adjacent voxels (using the same adjacency relation as when computing the DT) has a higher distance label. With a slight modification, this method can be used also for $W D T$; to avoid detection of false CMBs, the distance label 3 has to be changed to 1 when using the $W D T$. This is in analogy with the weighted distance transform in $\mathbb{Z}^{2}$ with weights 3 and 4, [8]. The local check to be performed is: $x$ is a CMB if it has a distance label strictly larger than all its neighbours minus the corresponding weight.

It is not enough to identify local maxima to extract CMBs in an EDT. To find the CMBs, a look-up table is created. For a center $x$ of a ball $B(x, r)$ with a given radius $r$, it is computed in advance how big the radii of the balls with centers at positions that are adjacent to $x$ have to be to cover the ball $B(x, r)$. The values are stored in the look-up table. To check if a voxel $x$ with distance label $r$ is a CMB, the distance labels of the neighbours to $x$ are compared with the values in the look-up table in Table 1 . Voxel $x$ is not a CMB if any neighbour has a distance label strictly greater than the value in the look-up table, otherwise it is a CMB. Observe that some distance labels are not considered in Table 1 since they cannot appear in a $E D T$ in $\mathbb{Z}^{3}$. This method, however, produces some false CMBs, at least for larger $r$, [9]. All the true CMBs are present and therefore the method is accurate enough for the classification in this paper. To only get the true CMBs, larger neighbourhood than $3 \times 3 \times 3$ must be used.

In Figure 3(a), a digitization of the elliptic cylinder in Figure 1(a) is shown. This object will be used as a running example throughout the paper. The sets of CMBs of the elliptic cylinder using the different metrics are shown in Figure 3(b)-(e).

From the set of CMBs, the original object can be reconstructed by using a reverse distance transform ( $\mathrm{rDT}$ ). A voxel belongs to the original object iff it is at a distance from a CMB less than the distance label of that CMB. For the $D^{6}, D^{26}$, and $<3,4,5>$ metrics, the $\mathrm{rDT}$ can be computed by an algorithm similar to the algorithm used for computing the DT. The CMBs are initially labeled with their distance label and non-CMB voxels are labeled zero. The distance values are propagated by assigning to each voxel the maximum value of its distance label and the distance label of the adjacent voxels minus the

Table 1. Look-up table with values of the radii for face-neighbours, flut, edgeneighbours, elut, vertex-neighbours, vlut corresponding to a voxel $x$ with distance label $r$. The first 21 entries in the look-up table are shown. The values in the table should be compared with the squared distance label

\begin{tabular}{|l|l|l|l|l|l|l|l|l|l|l|l|l|l|l|l|l|l|l|l|l|l|}
\hline$r^{2}$ & 1 & 2 & 3 & 4 & 5 & 6 & 8 & 9 & 10 & 11 & 12 & 13 & 14 & 16 & 17 & 18 & 19 & 20 & 21 & 22 & 24 \\
\hline flut & 2 & 5 & 6 & 7 & 10 & 11 & 12 & 14 & 17 & 18 & 19 & 19 & 21 & 22 & 26 & 27 & 28 & 28 & 30 & 31 & 31 \\
\hline elut & 3 & 6 & 9 & 10 & 11 & 14 & 15 & 19 & 20 & 21 & 22 & 23 & 26 & 27 & 27 & 30 & 33 & 34 & 35 & 36 & 37 \\
\hline vlut & 4 & 7 & 10 & 13 & 13 & 15 & 18 & 20 & 23 & 23 & 25 & 28 & 28 & 30 & 30 & 35 & 35 & 37 & 37 & 39 & 42 \\
\hline
\end{tabular}




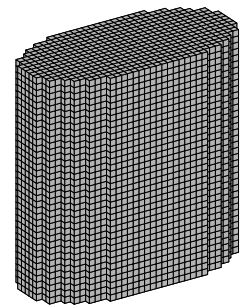

(a)

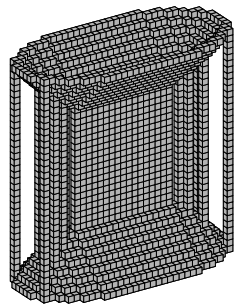

(b)

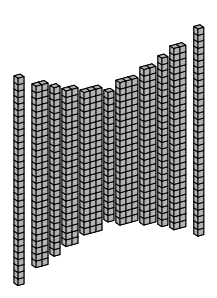

(c)

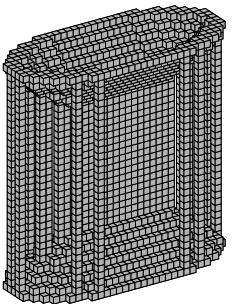

(d)

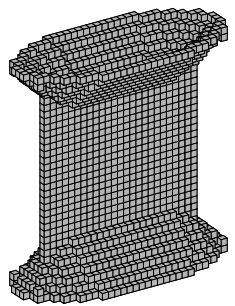

(e)

Fig. 3. CMBs of an elliptic cylinder (a) using (b) $D^{6}$, (c) $D^{26}$, (d) $<3,4,5>$, and (e) the Euclidean metrics

corresponding weight. When computing the rDT based on the Euclidean metric, e.g., the method described in [6] can be used.

\section{Classification of CMBs in $\mathbb{Z}^{3}$}

It is difficult to make an applicable definition of the order of tangency in $\mathbb{Z}^{3}$, due to its discrete structure. Therefore, this measure is not considered in the classification of CMBs in $\mathbb{Z}^{3}$. The number of tangencies with the object boundary can, however, be generalized to the digital space in a natural way.

In this classification, the notation $A_{m}^{n}$ will be used, where $m$ and $n$ denotes the following:

$m$ The number of tangency regions of the $\mathrm{CMB}$, i.e., the number of connected regions where the border of the $\mathrm{CMB}$ intersects the border of the object.

$n$ The number of non-tangency regions of the CMB, i.e., the number of connected regions where the border of the CMB is in the interior of the object.

In general, this measure can be approximated for a maximal ball $B(x, r)$ with centre $x$ and radius $r$ in an object $X$ by considering the number of connected components of the intersection of the object and the border of a ball with a slightly larger radius. The number of connected components of $\partial B(x, r+\epsilon) \cap X$ and $\partial B(x, r+\epsilon) \cap \bar{X}$ are considered for some sufficiently small tolerance $\epsilon>0$. By keeping the tolerance $\epsilon$ as small as possible, small variations in the border of $X$ will be detected. Since many different metrics are considered in this paper, finding the minimal $\epsilon$ such that $B(x, r+\epsilon) \backslash B(x, r)$ is a connected set is not trivial. Using $\epsilon=1$ will make $B(x, r+\epsilon) \backslash B(x, r)$ 26-connected for $D^{6}$ and 6 -connected for $D^{26}$. For the $\langle 3,4,5\rangle$ and the Euclidean metrics, general properties of the resulting set are not easily derived. Instead of being enlarged by increasing the radii, the balls will be dilated with the set $C=\left\{(x, y, z) \in \mathbb{Z}^{3}: \max (|x|,|y|,|z|) \leq 1\right\}$. In this way, an approach that is applicable on all metrics is achieved since using a dilation guarantees that $(B(x, r) \oplus C) \backslash B(x, r)$ is connected. 
If the thickness of the object at some point equals an even number of voxels, the set of CMBs might be two voxels thick in places, $[2,10]$. This is compensated for by allowing CMBs with the same distance label adjacent (using the same adjacency relation as when computing the DT and 26-adjacency for the EDT) to $x$ participate in the computation of $A_{m}^{n}$ for $x$.

In the present implementation, $B(x, r)$ is obtained by a $\mathrm{rDT}$. Since the radii are known, these computations can be restricted to a small subset of the image. In pseudocode, the algorithm is

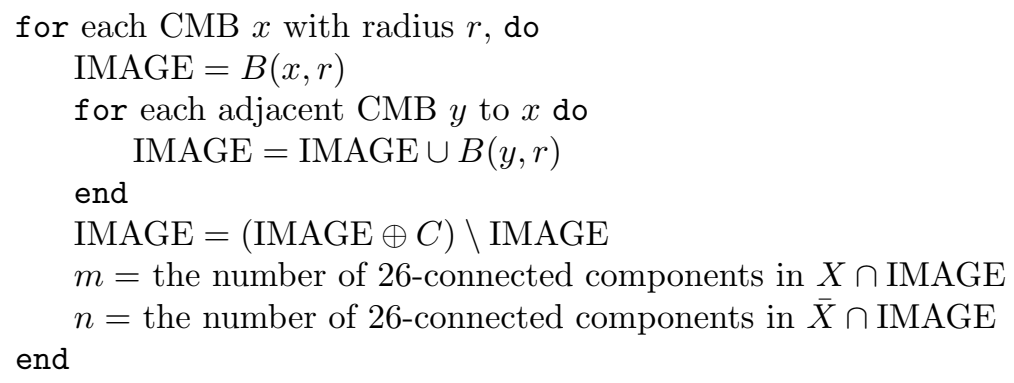

With each CMB labeled with values of $m$ and $n$, the classification of the CMBs into the sets $A_{m}^{n}$ is complete.

\section{$5 \quad$ Interpretation of the Classes}

A CMB that has two tangency regions and one non-tangency region corresponds to a surface-part of the skeleton. Such a CMB is surrounded by CMBs in directions where the tangencies do not occur. This corresponds to the situation in a local neighbourhood of a point in a surface. A ball with sufficiently small radius and centre in the interior of a surface will have two connected components in the background and one connected component in the surface. At the border of a surface a sufficiently small ball will have exactly one tangency region and one non-tangency region. A ball with centre at the intersection of surfaces has at least three tangency regions and one non-tangency region. With this reasoning, we get the following classification: A CMB classified as

- $A_{1}^{1}$ is located at a border of a surface or at an endpoint of a curve of the skeleton.

- $A_{2}^{1}$ belongs to a surface part of the skeleton.

- $A_{m}^{1}, m>2$ is located at the intersection curve of $m$ surfaces.

- $A_{1}^{2}$ belongs to a curve part of the skeleton.

- $A_{1}^{n}, n>2$ is located at the point of intersection between $n$ curves.

- $A_{m}^{n}, m, n>1$ is located at the intersection of both surfaces and curves.

See Figure 4 for a classification of the CMBs in Figure 3. 

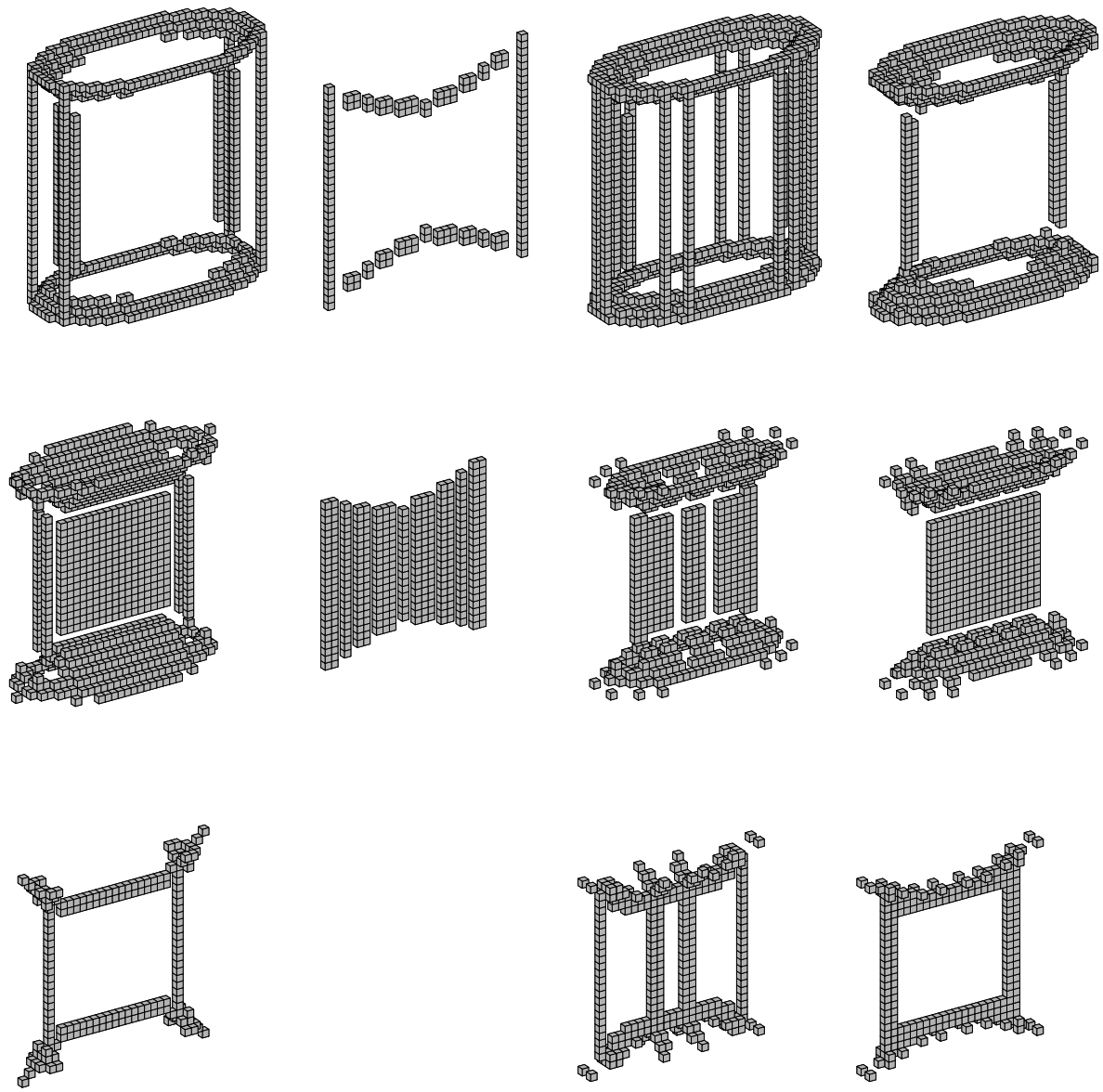

Fig. 4. Classification of the CMBs in Figure 3. Left to right: $D^{6}, D^{26},<3,4,5>$, and Euclidean metric.

Top row: $A_{1}^{1}$, second row: $A_{2}^{1}$, and bottom row: $A_{m}^{1}, m>2$

It is well-known that the set of CMBs is highly sensitive to noise (e.g., border voxels added to the object). This is not the case for the classification of CMBs into the sets corresponding to surfaces, i.e., $A_{m}^{1}, m \geq 2$ and $A_{m}^{n}, m, n>1$. A noise voxel might increase the number of non-tangency regions by one for some CMB. The CMB will, however, still be assigned to a surface of the skeleton, but with the value of $n$ increased by one. Often, a border voxel caused by noise will cause a single $\mathrm{CMB}$ close to the object border. Such a CMB in general belongs to $A_{1}^{1}$ and does not affect the surface-part of the skeleton. See Figure 5 . 

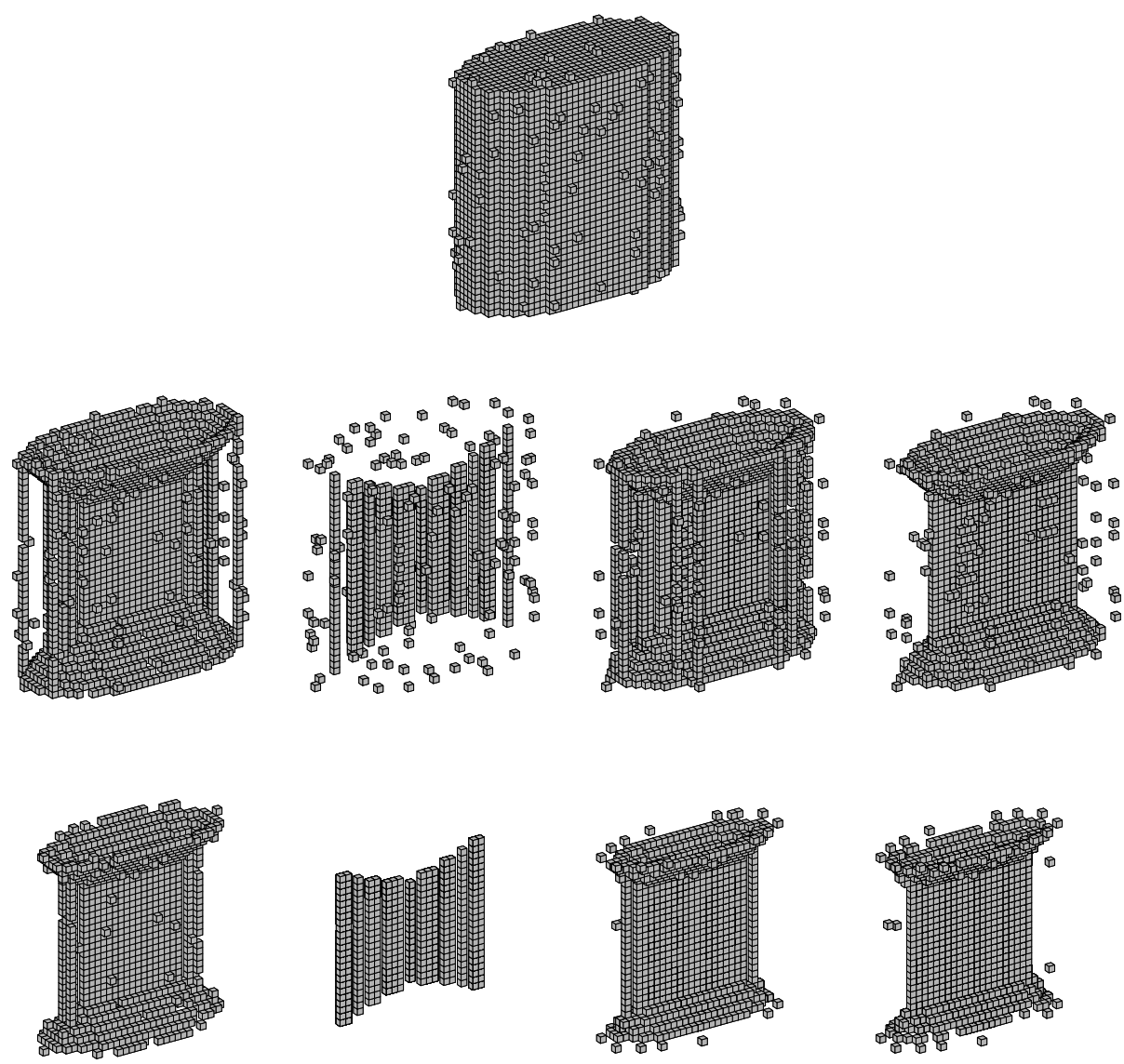

Fig. 5. Classification of the CMBs of a noisy object. Left to right: $D^{6}, D^{26},<3,4,5>$, and Euclidean metric.

Top row: The cylinder in Figure 3(a). Middle row: The set of CMBs. Bottom row: The union of $A_{m}^{1}, m \geq 2$ and $A_{m}^{n}, m, n>1$

\section{Conclusions and Future Work}

Each CMB is classified as belonging to a surface part, a curve part, the intersection of surfaces and/or curves, or the class $A_{1}^{1}$. The classification of CMBs is intended for the construction of $\mathbb{Z}^{3}$ skeletons. A CMB belonging to $A_{1}^{1}$ should either be assigned to the border of a surface of the skeleton or to the endpoint of a curve. The endpoint of a curve corresponds, in general, to a small detail or a noise voxel in the object resulting in a protrusion of the skeleton. Removing curve end-points corresponds to pruning, an important post-processing step in the skeletonization process, [10]. With the classification proposed in this paper, 
CMBs caused by noise or details at the border of the object can be handled consistently and will not cause unwanted surfaces of the skeleton.

Ideally, the skeleton of the running example would consist only of surfaces. By considering the union of $A_{m}^{1}, m \geq 2$ and the CMBs in $A_{1}^{1}$ that is located at the boundary of surfaces consisting of voxels in $A_{m}^{1}, m \geq 2$, the classes shown in Figure 4 with $D^{6},<3,4,5>$, and Euclidean metrics, will constitute sets that are close to the medial axis representation in $\mathbb{R}^{3}$, Figure $1(\mathrm{~b})$. Due to the shape of the balls using $D^{26}$, the surface representation using this metric is far from what was obtained in $\mathbb{R}^{3}$.

The main advantage with this classification is that, since the CMBs are divided into surfaces and curves, the rules to construct a connected skeleton from the set of CMBs can be constructed specifically for building surfaces or curves. The aim is to use this classification to construct skeletons consisting of surfaces and curves, where the two classes are well-defined.

\section{Acknowledgement}

Many thanks to Prof. Gunilla Borgefors and Dr. Stina Svensson, both Centre for Image Analysis, Uppsala, Sweden, for their valuable comments and suggestions during the development of the classification and preparation of the manuscript.

\section{References}

1. Blum, H.: A transformation for extracting new descriptors of shape. In WathenDunn, W., ed.: Proc. Models for the Perception of Speech and Visual Form, Cambridge, MA, MIT Press (1967) 362-380

2. Sanniti di Baja, G., Svensson, S.: Surface skeletons detected on the $D^{6}$ distance transform. In: Proceedings of the Joint IAPR International Workshops on Advances in Pattern Recognition, Springer-Verlag (2000) 387-396

3. Giblin, P., Kimia, B.B.: A formal classification of 3D medial axis points and their local geometry. IEEE Trans. Pattern Anal. Mach. Intell. 26 (2004) 238-251

4. Borgefors, G.: On digital distance transforms in three dimensions. Computer Vision and Image Understanding 64 (1996) 368-376

5. Ragnemalm, I.: The Euclidean distance transform in arbitrary dimensions. Pattern Recognition Letters 14 (1993) 883-888

6. Coeurjolly, D.: d-dimensional reverse Euclidean distance transformation and Euclidean medial axis extraction in optimal time. In: Proceedings of $11^{\text {th }}$ Conference on Discrete Geometry for Computer Imagery, Naples, Italy. (2003) 327-337

7. Rosenfeld, A., Pfaltz, J.L.: Sequential operations in digital picture processing. J. ACM 13 (1966) 471-494

8. Arcelli, C., Sanniti di Baja, G.: Finding local maxima in a pseudo-Euclidean distance transform. Computer Vision, Graphics, and Image Processing 43 (1988) 361-367

9. Remy, E., Thiel, E.: Look-up tables for medial axis on squared Euclidean distance transform. In: Proceedings of $11^{\text {th }}$ Conference on Discrete Geometry for Computer Imagery, Naples, Italy. (2003) 224-235

10. Svensson, S., Sanniti di Baja, G.: Simplifying curve skeletons in volume images. Computer Vision and Image Understanding 90 (2003) 242-257 